Copyright $\odot$ IFAC Artificial Intelligence in Real Time Control,

Budapest, Hungary, 2000

\title{
ON THE DYNAMIC INSTABILITY OF A CLASS OF SWITCHING SYSTEM
}

\author{
Robert Noel Shorten* Fiacre Ó Cairbre** \\ Paul Curran*** \\ * Department of Computer Science, National University of \\ Ireland, Maynooth,rshorten@cs.may.ie \\ ** Department of Mathematics, National University of Ireland, \\ Maynooth, foc@maths.may.ie \\ *** Department of Electronic and Electrical Engineering, National \\ University of Ireland, Dublin, paul.curran@ucd.ie
}

\begin{abstract}
A sufficient condition for the existence of a destabilising switching sequence for the system $\dot{x}=A(t) x, A(t) \in\left\{A_{1}, A_{2}, \ldots, A_{M}\right\}, A_{i} \in \mathbb{R}^{N \times N}$, where the $A_{i}$ are Hurwitz matrices, is that there exists non-negative real constants $\alpha_{1}, \alpha_{2}, \ldots, \alpha_{M}$, $\alpha_{i} \geq 0, \sum_{i=1}^{M} \alpha_{i}>0$, such that the matrix pencil $\sum_{i=1}^{M} \alpha_{i} A_{i}$ has at least one eigenvalue with a positive real part. An informal proof of this result based upon Floquet theory was presented in (Shorten, 1996; Shorten and Narendra, 1997). In this paper we present a rigourous basis for the proof of this result. Further, we use this result to identify several classes of linear switching systems, which admit the existence of a destabilising switching sequence. These systems provide insights into the relationship between the existence of a common quadratic Lyapunov function and the existence of a destabilising switching sequence for low order systems, as well as the robustness of a class of switching system that is known to be exponentially stable. Copyright $\odot 2000$ IFAC
\end{abstract}

Keywords: Stability Theory, Switching Systems, Hybrid Systems, Multiple Models

\section{INTRODUCTION}

Recent years have witnessed an enormous growth of interest in dynamic systems that are characterised by a mixture of both continuous and discrete dynamics. Such systems are referred to as switching systems or hybrid systems in the scientific literature, and are commonly found in many practical applications. Perhaps the most important consideration in the analysis of these systems is their stability. In spite of the vast amount of work currently in progress in this area, many fundamental questions related to their stability remain unanswered. The ultimate objective of the work presented in this paper is to address several open problems related to the stability of a particular class of hybrid systems. We consider systems described by the following equation,

$$
\dot{x}=A(t) x, A(t) \in\left\{A_{1}, A_{2}, \ldots, A_{M}\right\},
$$

where the $A_{i} \in \mathbb{R}^{N \times N}$ matrices are assumed to be Hurwitz (i.e. all eigenvalues have negative real parts). Equation (1) describes many of the most commonly studied multiple model systems, including the multiple model switching and tuning paradigm introduced in (Narendra and Balakrishnan, 1994), some gain scheduling systems (Leith and Leithead, n.d.), and some local model networks (Murray-Smith and Johanson, 1996). The stability of equation (1) has also been the subject of much attention in the stability community, and more recently in the hybrid systems community (Shorten and Narendra, 1998a). Typically the approach taken is to impose conditions on the $A_{i}$ matrices, or on the switching times, such that 
the time varying system (1) is asymptotically stable. Almost all of the classical results in the area have been derived in this manner including the Circle Criterion (Narendra and Taylor, 1973) (and other results based upon the existence of a common quadratic Lyapunov function), the Small Gain Theorem (Vidysagar, 1993) and results for slowly varying systems and other special systems (Desoer, 1969; Bellman, 1953). More recent attention in the control community has focussed upon the development of numerical algorithms for testing these conditions (typically using Linear Matrix Inequalities) (Boyd et al., 1994). Similarly, in the hybrid sytems community, attention has focussed on finding conditions that ensure stability. Again, the principal results in this area, namely the concepts of multiple Lyapunov functions (Branicky, 1994), and of piecewise quadratic Lyapunov functions (Johansson, 1996), have also been based upon the concept of determining entire classes of hybrid systems that are asymptotically stable. Good overviews of the classical work in stability theory can be found in (Curran, 1998; Vidyasagar, 1986; Willems, 1976), and much of the more recent work is described in (Liberzon and Morse, 1999).

The approach taken in this paper is quite different. Here we attempt to classify possible instability mechanisms in systems decribed by Equation (1). This is similar to the Describing Function (Mees and Bergen, 1975) approach where instability is assumed to occur in the form of a sinusoidal limit cycle. In this paper we consider several classes of systems, of the form of Equation (1), which become unstable as a result of an unstable 'chattering-like' mechanism.

\section{MATHEMATICAL RESULTS}

In this section we present a matrix pencil result that characterises a particular instability mechanism in the system (1). This result is based upon a simple observation in the phase plane; namely that if a point $x_{0}$ in the state space exists, such that $\left(\sum_{i=1}^{M} \alpha_{i} A_{i}\right) x_{0}=k x_{0},, k>0$, then it is intuitively possible, by an appropriate choice of switching, for the solution to the switching system (1) to become unbounded. This observation is depicted in Figure 1 for second order systems with $M=2$.

Although this observation is trivial, and has almost certainly been observed by other researchers (Angeli, 1999), its proof is non-trivial and is based upon a theorem from eigenvalue perturbation theory (Lancaster and Tismenetsky, 1985; Kato, 1980). For ease of exposition the relevant

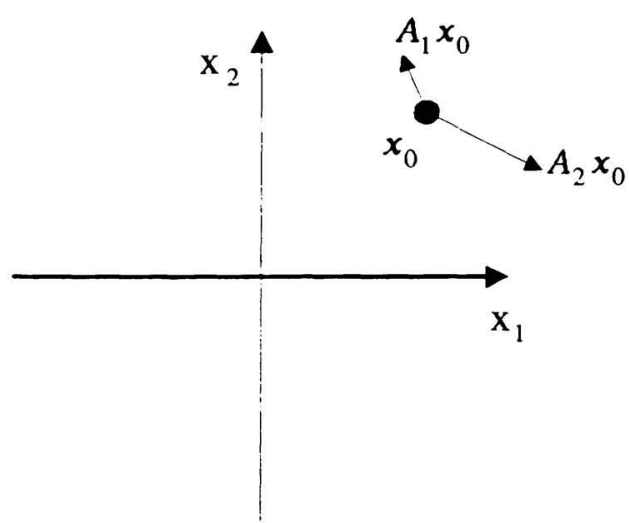

Fig. 1. Geometrical motivation of Theorem 2.2

mathematical theorem is quoted from (Lancaster and Tismenetsky, 1985), and brief comments regarding its use in our context are provided. We begin with the definition of a matrix pencil.

The matrix pencil $\sigma\left[A_{i}, M\right]$ : The pencil $\overline{\sum_{i=1}^{M} \alpha_{i} A_{i}, \alpha_{i} \geq 0, \sum_{i=1}^{M} \alpha_{i}>0}$, is used in the sequel. In view of this we shall use the notation

$$
\sum_{i=1}^{M} \alpha_{i} A_{i}, \alpha_{i} \geq 0, \sum_{i=1}^{M} \alpha_{i}>0 \triangleq \sigma\left[A_{i}, M\right]
$$

and refer to $\sigma\left[A_{i}, M\right]$ as a matrix pencil. If the eigenvalues of $\sigma\left[A_{i}, M\right]$ are in the open lefthalf of the complex plane, denoted $\mathbb{C}^{-}, \forall \alpha_{i} \geq$ $0, \sum_{i=1}^{M} \alpha_{i}>0$, then we shall refer to $\sigma\left[A_{i}, M\right]$ as a strictly Hurwitz pencil. A non-strictly Hurwitz pencil whose eigenvalues lie in the closed left-half of the complex plane $\forall \alpha_{i} \geq 0 \sum_{i=1}^{M} \alpha_{i}>0$, i.e. some of the eigenvalues have real parts that are zero, is referred to as a marginally Hurwitz pencil.

The following result is crucial to the discussion in the sequel.

Theorem 2.1. (Lancaster and Tismenetsky, 1985) Let $A(\zeta)=\sum_{n=0}^{\infty} \zeta^{n} A^{(n)}$ be a matrix that is analytic in $\zeta$ on a neighbourhood of $\zeta=0$ and suppose that $A(0)=A$. Let $\lambda$ be an eigenvalue of $A$ of index 1 , and of algebraic multiplicity $\mathrm{m}$, and let $\lambda_{j}(\zeta)$ be an eigenvalue of $A(\zeta)$ for which $\lambda_{j}(0)=\lambda$. Let $Z$ be the idempotent component matrix of $\lambda$. Then there is a number $a_{j}$, and a positive integer $l \leq m$ such that

$$
\lambda_{j}(\zeta)=\lambda+a_{j} \zeta+O\left(|\zeta|^{1+\frac{1}{l}}\right)
$$

as $|\zeta| \rightarrow 0$, and $a_{j}$ is an eigenvalue of $Z A^{(1)} Z$.

Theorem 2.2. A sufficient condition for the existence of a switching sequence, such that the system (1) is unstable, is that the there exists nonnegative constants $\left\{\alpha_{1}, \alpha_{2}, \ldots, \alpha_{M}\right\}$, such that the matrix pencil $\sigma\left[A_{i}, M\right]$ has an eigenvalue with a positive real part. 
Proof : In order to demonstrate the existence of an unstable switching sequence for Equation (1), it is sufficient to show the existence of a periodic switching sequence for which the the state of the system becomes unbounded.

Consider the system (1) with $A(t)=A(t+$ $T)$ for all $t>0$, where $T$ is the fixed period. Further, let the matrices $\left\{A_{1}, A_{2}, \ldots, A_{M}\right\}$ be indexed according to the order in which they describe the system dynamics, and let the M-tuple $\left\{t_{1}, t_{2}, \ldots, t_{M}\right\}$ describe the time for which each of the matrices describes the system dynamics, i.e., $\dot{x}=A_{j} x$, describes the evolution of the system dynamics for $\left(\sum_{i=1}^{j-1} t_{i}\right) \leq t \leq\left(\sum_{i=1}^{j} t_{i}\right)$. The solution to Equation (1) at time $T=\sum_{i=1}^{M} t_{i}$, is given by,

$$
\begin{aligned}
x(T) & =\left(e^{A_{2} t_{1}} e^{A_{,} t_{2}} \ldots e^{A_{M} t_{M}}\right) x_{0}, \\
& =\Phi(T) x_{0}
\end{aligned}
$$

where $x_{0} \in \mathbb{R}^{N}$ is some initial condition. A sufficient condition for instability of $(1)$ is that the matrix $\Phi(T)$ has an eigenvalue whose magnitude is greater than unity (Mohler, 1991). Equation (4) can be expanded as a power series,

$$
\begin{aligned}
\Phi(T) & =I+\left(\sum_{i=1}^{M} \alpha_{i} A_{i}\right) T+K_{2} T^{2}+\ldots \\
& =I+\sigma\left[A_{i}, M\right] T+\ldots
\end{aligned}
$$

where $\alpha_{i}=\frac{t_{1}}{T}$, and where $h_{2} \in \mathbb{R}^{N \times N}$. The expansion (5) is the product of $M$ absolutely convergent series, and is analytic in $T$. Hence, from Theorem 2.1 and (Kato, 1980) (page 81), it follows that, given any eigenvalue $a_{j}$ of $\sigma\left[A_{i}, M\right]$, an eigenvalue of $\Phi(T)$ can be written,

$$
\lambda_{j}(T)=1+a_{j} T+f(T),
$$

where $f(T)=O\left(T^{\beta}\right), \beta>1$, as $T \rightarrow 0$. Thus, there exist constants $C, \epsilon$ such that

$$
|f(T)| \leq C T^{\beta}, \text { for } 0<T<\epsilon .
$$

Hence, $|\Re(f(T))| \leq C T^{\beta}$ for $0<T<\epsilon$, where $\Re(z)$ denotes the real part of a complex number $z$. Now consider the case when the $j^{\text {th }}$ eigenvalue, $a_{j}$, of the matrix $\sigma\left[A_{i}, M\right]$, has a positive real part for some $\left\{\alpha_{1} \ldots, \alpha_{M}\right\}, \alpha_{i} \geq 0, \sum_{i=1}^{M} \alpha_{i}>0$. Hence, there exists $\delta<\epsilon$, such that for $0<T<\delta$. we have the following:

$$
\begin{aligned}
& C T^{3}<\Re\left(a_{j}\right) T, \\
&|\Re(f(T))|<\Re\left(a_{j}\right) T, \\
& \Re\left(a_{j}\right) T+\Re(f(T))>0, \\
& \Re\left(1+a_{j} T+f(T)\right)>1, \\
& \Re\left(\lambda_{j}(T)\right)>1, \quad \text { Q.E.D. }
\end{aligned}
$$

Comment: Precise definitions and a discussion of the idempotent component matrices of a matrix $A$ can be found in (Lancaster and Tismenetsky, 1985).

Comment : In the case where $\sigma\left[A_{i}, M\right]$ is marginally Hurwitz, then we say that a marginally unstable switching sequence exists for the system (1)

Comment : Using the notation of Theorem 2.1, the following statements can be easily shown for $\Phi(T)$ :
(a) : $A(0)=\mathrm{I}$;
(b) : $\lambda=1$ is an eigenvalue of $A(0)$ of index 1 ;
(c) : $A^{(1)}=\sigma\left[A_{i}, M\right]$;
(d) : The idempotent matrix of $\lambda=1$ is given by $Z=I$.

Comment : For the instability mechanism described by Theorem 2.2 , it follows that the system is unstable for all $T>T_{\text {crit }}$. This is in contrast to other instability mechanisms (those charactersied by describing function analysis), where the system is unstable for a range of switching times

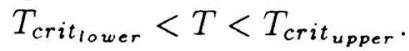

Comment : For second order switching systems, it follows that $\operatorname{Trace}\left(\sigma\left[A_{i}, M\right]\right)<0$. Hence, for unstable switching systems of the type characterised by Theorem 2.2 , it follows that there exists $\left\{\alpha_{1}, \ldots, \alpha_{M}\right\}, \alpha_{i} \geq 0, \sum_{i=0}^{M} \alpha_{i}>0$, such that $\operatorname{det}\left\{\sigma\left[A_{i}, M\right]\right\}=\overline{0}$. Hence, the pencil $\sigma\left[A_{i}, M\right]$ cannot have purely imaginary eigenvalues.

Comment: The work by Feron that is referred to in (Liberzon and Morse, 1999), and the work described in (Angeli, 1999) is closely related to the above result.

\section{COMMENTS AND EXAMPLES}

The result in Theorem 2.2 has important consequences for switching systems described by Equation (1). Here we briefly list some of these.

\subsection{General switching systems}

A function of the form $V(x)=x^{T} P x, P=P^{T}>$ $0, \quad P \in \mathbb{R}^{N \times N}$, with the property that its time derivative along any trajectory of the system (1) is negative definite, or alternatively that,

$$
A_{i}^{T} P+P A_{i}=-Q_{i}, i \in\{1,2, \ldots M\},
$$

where the matrices $Q_{i}$ are symmetric and positive definite, is a common quadratic Lyapunov function (CQLF) for the dynamic systems,

$$
\Sigma_{A_{1}}: \dot{x}=A_{i} x, i \in\{1,2, \ldots M\} \text {, }
$$


where $A_{i} \in \mathbb{R}^{N \times N}$. The existence of such a function is sufficient to demonstrate the asymptotic stability of (1). It is well known that a necessary condition for the existence of a common quadratic Lyapunov function for systems of the form of (1), is that the pencil $\sigma\left[A_{i}, M\right]$ is strictly Hurwitz. Further, Theorem 2.2 also implies that if this pencil is not strictly Hurwitz, and has an eigenvalue with a positive real part for some $\left\{\alpha_{1}, \ldots, \alpha_{M}\right\}, \alpha_{i} \geq 0$, $\sum_{i=0}^{M} \alpha_{i}>0$, then the system (1) is unstable. Hence, the condition that the pencil $\sigma\left[A_{i}, M\right]$ is strictly Hurwitz, or is marginally Hurwitz, is necessary for the stability of Equation (1).

\subsection{Second order switching systems and common quadratic Lyapunov functions}

A necessary and sufficient condition for the existence of a CQLF for the system,

$$
\dot{x}=A(t) x, \quad A(t) \in\left\{A_{1}, A_{2}\right\},
$$

where the $A_{i}, i \in\{1,2\}$, are constant Hurwitz matrices in $\mathbb{R}^{2 \times 2}$ is that the matrix pencils,

$$
\begin{array}{r}
\alpha A_{1}+(1-\alpha) A_{2}, \alpha \in[0,1], \\
\alpha A_{1}+(1-\alpha) A_{2}^{-1}, \alpha \in[0,1],
\end{array}
$$

are both Hurwitz(Shorten and Narendra, 1999; Cohen and Lewkowicz, 1991). It follows from the arguments in Theorem 2.2 that the non-existence of a CQLF for (10) implies that an unstable, or a marginally unstable switching sequence exists for at least one of the dual switching systems

$$
\begin{aligned}
& \dot{x}=A(t) x, \quad A(t) \triangleq\left\{A_{1}, A_{2}\right\}, \\
& \dot{x}=A(t) x, \quad A(t) \triangleq\left\{A_{1}, A_{2}^{-1}\right\}
\end{aligned}
$$

Although this observation is not true for $M>2$ matrices (Shorten and Narendra, 2000), it is somewhat surprising since it implies a type of equivalence between the stability problem for (10), and the CQLF existence problem for simple second order switching systems with $M=2$. This is contrary to the commonly held view that the requirement that a CQLF exists, places unnecessarily conservative restrictions on the switching system. Further, this observation provides an exact indication of the conservatism (non-conservatism) of the CQLF approach to proving asymptotic stability for second order systems, as well as providing valuable insights into the conservatism of the CQLF approach for higher order systems.

\subsection{Matrices that are simultaneously triangularisable}

Much recent work has focussed on systems of the form of Equation (1) where the matrices
$\left\{A_{1}, \ldots, A_{M}\right\}$ can be simultaneously triangularised (have property $P$ (Laffey, 1978)), i.e. there exists a non-singular transformation $T$ such that the matrices $\left\{T A_{1} T^{-1}, \ldots, T A_{M} T^{-1}\right\}$ are simultaneously upper triangular. It has been shown by several authors that a CQLF exists in such circumstances, and that the switching system (1) is therefore asymptotically stable (Mori et al., 1996; Mori et al., 1997; Liberzon et al., 1998; Shorten and Narendra, 1998b; Cohen et al., 1997). This result is not surprising since the exponential stability of (1) follows directly from its solution when the $A_{i}$ matrices, $i \in\{1, \ldots, M\}$ are upper triangular. Despite the benign nature (from a stability viewpoint) of such systems, their robustness properties prove to be interesting. In this section we show, by means of a simple example, that stability results based upon simultaneous triangularisation are, via Theorem 2.2, not robust. We then briefly consider the generic stability of a related class of system.

3.3.1. Robustness : Consider the dynamic system (1), where the matrices $\left\{T A_{1} T^{-1}, T A_{2} T^{-1}\right\}$, $A_{i} \in \mathbb{R}^{2 \times 2}$ are diagonal matrices (a special case of simultaneous triangularisation). This implies that $A_{1}$ and $A_{2}$ have two common eigenvectors. For convenience, let these eigenvectors be $v_{1}=[0,1]^{T}$ and $v_{2}=[1,0]^{T}$. We consider perturbing the eigenvectors of $A_{2}$ as depicted in Figure 2, where $L$ is some positive real number.

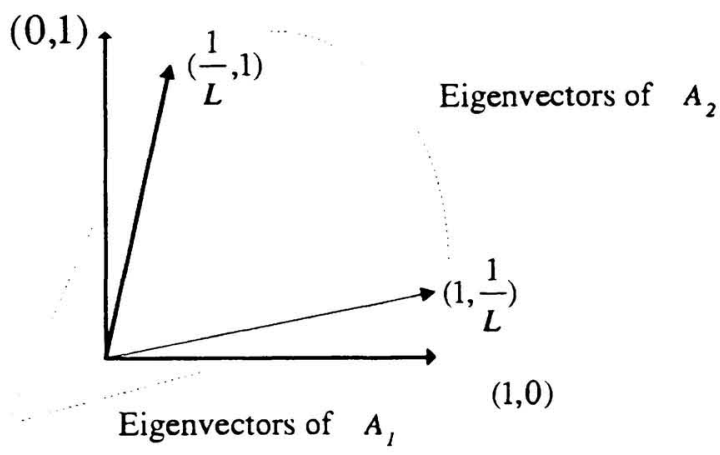

Fig. 2. Geometrical construction of eigenvectors

Let,

$$
\begin{aligned}
& A_{1}=\left(\begin{array}{cc}
-K & 0 \\
0 & -\frac{1}{L^{2}}
\end{array}\right), \\
& A_{2}=M\left(\begin{array}{cc}
-K & 0 \\
0 & -\frac{1}{L^{2}}
\end{array}\right) M^{-1},
\end{aligned}
$$

with 


$$
M=\left(\begin{array}{cc}
1 & \frac{1}{L} \\
\frac{1}{L} & 1
\end{array}\right)
$$

where both $L$ and $K$ are real positive numbers. The matrices $A_{1}$ and $A_{2}$ satisfy the following properties:

(a) $A_{1}$ and $A_{2}$ have identical eigenvalues;

(b) $\lim _{L \rightarrow \infty} A_{1}=\lim _{L \rightarrow \infty} A_{2}$.

It would not be unreasonable to expect, in view of properties (a) and (b) above, that switching systems of the form of Equation (1), with $A_{1}$ and $A_{2}$ defined as in (15) and (16), and with $M=2$ are stable, provided $L$ is large enough. However, this is not true, as verified by the following theorem.

Theorem 3.1. Consider the switching system (1), with $M=2$, and with $A_{1}$ and $A_{2}$ defined in Equations (15) and (16) respectively. Then for any $L>1$, there exists $K$ such that the system (1) is unstable for some periodic switching sequence.

Proof: Let $L>1$. Then it follows from algebraic arguments that one eigenvalue of $A_{1}+A_{2}$ has a real part that is positive for a sufficiently large $K$. Hence, from Theorem 2.2, a switching sequence exists such that the solution to system (1) is unbounded.

Comment: Theorem 3.1 implies that two Hurwitz matrices, whose eigenvectors are arbitrarily close to each other, can be used to construct an unstable switching system. This observation has important consequences for the robustness of results based upon simultaneously triangularisable matrices.

3.3.2. Extensions: In this section we briefly discuss the stability properties of a class of switching system that was introduced in (Shorten and Ó Cairbre, 2000a). In this paper, systems were considered where no single matrix $T$ exists that simultaneously transforms each $A_{i} \in \mathcal{A}$ to upper triangular form, but where a set of nonsingular matrices $T_{i j}$ exist such that the matrices $\left\{T_{i j} A_{i} T_{i j}^{-1}, T_{i j} A_{j} T_{i j}^{-1}\right\}, i, j \in\{1, \ldots, m\}$, are upper triangular. It was demonstrated that in general, a CQLF does not exist for such systems. Further, by imposing restrictions on the matrices $\left\{A_{1}, \ldots, A_{M}\right\}$, global attractivity of the origin could be shown. It was also conjectured that such systems might be in general asymptotically stable. We comment briefly here that this is not true. Simple examples can be generated that satisfy the conditions of Theorem 2.2, and are therefore unstable. Full details of one such example can be found in (Shorten and Ó Cairbre, 2000b).

\section{CONCLUDING REMARKS}

In this paper we have presented a simple result that characterises one particular instability mechanism for a class of switching system. This result has profound consequences for other classes of systems, and motivates a number of interesting research questions that relate to known classical stability results. One of the main objectives of the European Union funded research trianing network, Multi-Agent Control is to investigate some of these open questions, and to characterise other instability mechanisms in systems described by Equation (1).

\section{ACKNOWLEDGEMENTS}

This work was partially supported by the European Union funded research training network Multi-Agent Control, HPRN-CT-1999-00107. The first author gratefully acknowledges many discussions with Professor K.S. Narendra relating to the material presented in this paper.

\section{REFERENCES}

Angeli, D. (1999). A note on the stability of arbitrarily switched homogeneous systems. Submitted to systems and control letters.

Bellman, R. (1953). Stability Theory of Differential Equations. McGraw-Hill.

Boyd, S., L. El Ghaoui, E. Feron and V. Balakrishnan (1994). Linear Matrix Inequalities in System and Control Theory. SIAM.

Branicky, M.S. (1994). Stability of Switched and Hybrid Systems. In: Proceedings of Conference on Decision and Control, Florida, pp. 3498-3503.

Cohen, N. and I Lewkowicz (1991). Robust quadratic stability and convex invertible cones of matrices. Technical report. Technion, EE-797.

Cohen, N., I. Lewkowicz and L. Rodman (1997). Exponential stability of triangular differential inclusion systems. Systems and Control letters 30, 159-164.

Curran, P. F. (1998). An overview of absolute stability theory. In: Proceedings of 6th International Workshop on Nonlinear Dynamics of Electronic Systems.

Desoer, C.A. (1969). Slowly Varying System $\dot{x}=A(t) x$. IEEE Transactions on Automatic Control 14(6), 780-781.

Johansson, $M$. and Rantzer, A. (1996). Computation of piecewise quadratic lyapunov functions for hybrid systems, ISRN LUTFD2/TFRT-7459-SE. Technical report. Department of Automatic Control, Lund University, Sweden. 
Kato, T. (1980). Perturbation theory for linear operators. Springer.

Laffey, T.J. (1978). Simultaneous Triangularisation of Matrices - Low Rank Cases and the Nonderogatory Case. Linear and Multilinear Algebra 6(1), 269-305.

Lancaster, P. and H. Tismenetsky (1985). The Theory of Matrices. Academic Press.

Leith. D. J. and W. E. Leithead (n.d.). Survey of Gain Scheduling Analysis and Design. International Journal of Control (in press).

Liberzon, D. and A. S. Morse (1999). Basic problems in stability and design of switched systems. IEEE control systems magazine pp. 5970 .

Liberzon, Daniel, Joao P. Hespanha and S. Morse (1998). Stability of Switched Linear Systems: A Lie Algebraic Condition. Technical report. Laboratory for Control Science and Engineering, Yale University.

Mees, A.I. and A.R. Bergen (1975). Describing Functions Revisited. IEEE Transactions on Automatic Control 20(4), 473-478.

Mohler, R.R. (1991). Nonlinear Systems: Dynamics and Control. Prentice Hall.

Mori, Y., T. Mori and Y. Kuroe (1996). Classes of Systems Having Common Quadratic Lyapunov Functions: Triangular System Matrices. In: Proceedings of Electronic Information and Systems Conference (in Japanese).

Mori, Y., T. Mori and Y. Kuroe (1997). A Solution to the Common Lyapunov Function Problem for Continuous Time Systems. In: Proceedings of 36th Conference on Decision and Control.

Murray-Smith, R. and T.A. Johanson (1996). Multiple Model Approaches to Modelling and Control, editors R. Murray-Smith, T.A. Johansen. Taylor \& Francis.

Narendra, K and J Balakrishnan (1994). Intelligent Control using Fixed and Adaptive Models. In: Proceedings of $33 r d$ Conference on Decision and Control, pp. 1680-1685, Florida.

Narendra, K.S and J.H. Taylor (1973). Frequency Domain Criteria for Absolute Stability. Academic Press.

Shorten, R. (1996). A Study of Hybrid Dynamical Systems with Application to Automotive Control. PhD thesis. Department of Electrical and Electronic Engineering, University College Dublin, Republic of Ireland.

Shorten, R. and F. Ó Cairbre (2000a). A proof of Global Attractivity for a Class of Switching Systems using a non-lyapunov approach. Submitted to IMA Journal of Control and Optimisation.

Shorten, R. and F. Ó Cairbre (2000b). A proof of Global Attractivity for a Class of Switching Systems using a non-lyapunov approach part ii. In preparation.
Shorten, R. and K Narendra (1999). Necessary and Sufficient Conditions For The Existence Of A Common Quadratic Lyapunov Function For Two Stable Linear Second Order Systems. In: Proceedings of American Control Conference.

Shorten, R. and K Narendra (2000). Necessary and Sufficient Conditions For The Existence Of A Common Quadratic Lyapunov Function For M-Stable Linear Second Order Systems. In: Proceedings of American Control Conference.

Shorten, R. and K.S. Narendra (1997). A Sufficient Condition for the Existence of a Common Lyapunov Function for Two Second Order Systems: Part 1.. Technical report. Center for Systems Science, Yale University.

Shorten, R. and K.S. Narendra (1998a). Neccessary and Sufficient Conditions for the Existence of a Common Lyapunov Function for Two Second Order Systems. Technical report. Center for Systems Science.

Shorten, R and K.S. Narendra (1998b). On the Stability and Existence of Common Lyapunov Functions for Linear Stable Switching Systems. In Proceedings of Conference on Decision and Control, Florida Dec 15th- 18th.

Vidyasagar, M (1986). New directions of research in nonlinear system theory. Proceedings of the IEEE 74(8), 1060 - 1091.

Vidysagar, M. (1993). Nonlinear Systems Analysis. Prentice Hall.

Willems, J (1976). Mechanisms for the Stability and Instability in Feedback Systems. Proceedings of the IEEE 64(1), 24-35. 